\title{
Fecal Microbiome and Food Allergy in Pediatric Atopic Dermatitis: A Cross-Sectional Pilot Study
}

\author{
Karin B. Fieten ${ }^{a, e}$ Joan E.E. Totté ${ }^{c}$ Evgeni Levin ${ }^{d}$ Marta Reyman $^{a}$ \\ Yolanda Meijer $^{b}$ André Knulst ${ }^{a}$ Frank Schuren ${ }^{d}$ Suzanne G.M.A. Pasmans ${ }^{a}$ \\ ${ }^{a}$ Department of Dermatology and Allergology, University Medical Center Utrecht, and ${ }^{b}$ Department of Pediatric \\ Pulmonology and Allergology, Wilhelmina Children's Hospital, University Medical Center Utrecht, Utrecht, \\ 'Department of Dermatology, Erasmus MC University Medical Center Rotterdam, Rotterdam, and dTNO,

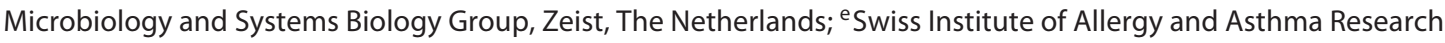 \\ (SIAF), University of Zürich, Davos, Switzerland
}

\section{Keywords}

Pediatric atopic dermatitis · Food allergy · Fecal

microbiome $\cdot$ Intestinal microbiota $\cdot$ Microbes

\begin{abstract}
Background: Exposure to microbes may be important in the development of atopic disease. Atopic diseases have been associated with specific characteristics of the intestinal microbiome. The link between intestinal microbiota and food allergy has rarely been studied, and the gold standard for diagnosing food allergy (double-blind placebo-controlled food challenge [DBPCFC]) has seldom been used. We aimed to distinguish fecal microbial signatures for food allergy in children with atopic dermatitis (AD). Methods: Pediatric patients with $A D$, with and without food allergy, were included in this cross-sectional observational pilot study. AD was diagnosed according to the UK Working Party criteria. Food allergy was defined as a positive DBPCFC or a convincing clinical history, in combination with sensitization to the relevant food allergen. Fecal samples were analyzed using $16 \mathrm{~S}$
\end{abstract}

rRNA microbial analysis. Microbial signature species, discriminating between the presence and absence food allergy, were selected by elastic net regression. Results: Eighty-two children with $A D$ ( 39 girls) with a median age of 2.5 years, and 20 of whom were diagnosed with food allergy, provided fecal samples. Food allergy to peanut and cow's milk was the most common. Six bacterial species from the fecal microbiome were identified, that, when combined, distinguished between children with and without food allergy: Bifidobacterium breve, Bifidobacterium pseudocatenulatum, Bifidobacterium adolescentis, Escherichia coli, Faecalibacterium prausnitzii, and Akkermansia muciniphila (AUC 0.83, sensitivity 0.77 , specificity 0.80 ). Conclusions: In this pilot study, we identified a microbial signature in children with $A D$ that discriminates between the absence and presence of food allergy. Future studies are needed to confirm our findings.

(c) 2018 The Author(s)

Published by S. Karger AG, Basel

Edited by: J. Gutermuth, Brussels.

\section{KARGER}

E-Mail karger@karger.com www.karger.com/iaa

\section{The Author(s) \\ Published by S. Karger AG, Basel \\ Karger \\ Open access}

This article is licensed under the Creative Commons AttributionNonCommercial-NoDerivatives 4.0 International License (CC BY NC-ND) (http://www.karger.com/Services/OpenAccessLicense). Usage and distribution for commercial purposes as well as any distribution of modified material requires written permission.
Correspondence to: Prof. Suzanne G.M.A. Pasmans

Department of Dermatology, Sophia Children's Hospital Erasmus University Medical Center Rotterdam

Wytemaweg 80, NL-3015 CN Rotterdam (The Netherlands)

E-Mail s.pasmans@erasmusmc.nl 


\section{Introduction}

The worldwide prevalence of atopic disease has been increasing in recent decades [1]. There is no clear reason for this observed increase in prevalence, but reduced early-life exposure to different microbes is thought to be a contributing factor [2-4]. Microbial colonization of the human intestine during infancy is important for the maturation of the immune system $[5,6]$. Intestinal microbiota can regulate metabolic and inflammatory responses and also modulate changes in the intestinal barrier. Several studies have shown associations between the intestinal microbiota and the subsequent development of atopic disease, including atopic dermatitis (AD), asthma, or rhinitis. However, few studies have investigated a specific link between the patterns of intestinal microbiota and food allergy. Furthermore, the gold standard for diagnosing food allergy (double-blind placebo-controlled food challenge [DBPCFC]) has rarely been used [7].

The microbiome can be considered a complex ecosystem where various species interact and group-based correlations have been identified [5]. Therefore the symbiosis of the different bacterial species and their patterns should be taken into account in data analysis. To be able to identify individual species and take the existing structures within the microbiome into account, advanced statistical modeling techniques are needed. Furthermore, the assessment of microbial diversity with molecular sequencing techniques, as opposed to culture-based techniques, reveals greater diversity and has shown the importance of uncultured species [8].

We hypothesized that children have distinct microbial patterns in their fecal microbiome that are associated with a clinical diagnosis of food allergy. In this cross-sectional pilot study, we aimed to identify microbial species in children with $\mathrm{AD}$, using $16 \mathrm{~S}$ rRNA microbial analysis followed by the statistical elastic net regression approach.

\section{Methods}

\section{Study Design and Participants}

Children with AD who were treated in the outpatient clinic of the Wilhelmina Children's Hospital of the University Medical Center Utrecht participated in this cross-sectional pilot study. Inclusion criteria were: a diagnosis of $\mathrm{AD}$, an age of $0-18$ years, parental ability to answer Dutch questionnaires, and the availability of a fecal sample for microbiome analysis. All study participants participated in a randomized controlled trial that compared shared medical appointments with individual consultations (ISRCTN08506572). The medical ethical committee of the University Medical Center Utrecht approved the study and written in- formed consent was obtained for all participants. Clinical history and serum samples were taken on the same day, fecal samples were provided within the next days, and a DBPCFC was planned to take place within months.

\section{Assessment of $A D$ and Food Allergy}

$\mathrm{AD}$ was diagnosed according to the criteria of Williams et al. [9]. $\mathrm{AD}$ severity was estimated using the self-administered eczema area and severity index (SA-EASI) by the research nurse [10]. Sensitization was determined by the level of specific IgE against common food allergens (hen's egg, cow's milk, peanut, hazelnut, fish, wheat, and soy). Both total and specific IgE were measured according to the manufacturer's protocol (Phadia, Uppsala, Sweden). A diagnosis of asthma or allergic rhinitis was based on the clinical history.

Food allergy was defined as a positive DBPCFC or a convincing clinical history, in combination with sensitization to a specific food, or, in the case of peanut allergy, a sensitization to Ara h 2 above the defined cut-off level in our clinic (5.17 kU/L) [11]. A convincing clinical history was defined as a reported type I allergic reaction with acute symptoms within $2 \mathrm{~h}$ after the ingestion of the food. DBPCFC was considered positive and was then terminated when persistent objective symptoms occurred (e.g., vomiting, generalized urticaria, wheezing, or a significant drop in blood pressure), or after subjective symptoms (oral allergy symptoms, nausea, and abdominal discomfort) on 3 subsequent doses, or the occurrence of a severe subjective symptom (abdominal pain/nausea with discomfort) lasting for $>45 \mathrm{~min}$, according to the international protocol [12]. Late reactions were assessed using follow-up by telephone the next day.

\section{Fecal Samples}

Fecal samples were collected at home and sent to the laboratory using the regular postal service. The samples were aliquoted and frozen at $-20^{\circ} \mathrm{C}$ for further processing.

\section{Fecal DNA Isolation}

Approximately $150 \mathrm{mg}$ of fecal material was directly transferred to the DNA isolation plate. Next, $0.5 \mathrm{~mL}$ phenol solution ( $\mathrm{pH}$ 8.0; catalogue P4557, Sigma-Aldrich, St Louis, MO, USA) was added and the cells in the samples were mechanically disrupted by bead-beating twice for 3 min with a 96-well-plate BeadBeater (Biospec Products, Bartlesville, OK, USA). Samples were centrifuged at $4,000 \mathrm{rpm}$ for $10 \mathrm{~min}$ to separate the aqueous and phenolic phases. The aqueous phase was transferred to a 96-well plate, and DNA was purified with the AGOWA mag Mini DNA isolation kit (AGOWA, LGC Genomics, Berlin, Germany) in accordance with the manufacturer's recommendations. After elution, the total bacterial load in each sample was assessed by quantitative PCR using a universal bacterial primer-probe set [13].

\section{$16 S$ rDNA Illumina Sequencing}

Analysis of the fecal microbiome composition was performed by mass sequencing of the V4 hypervariable region of the $16 \mathrm{~S}$ rRNA gene on the Illumina MiSeq sequencer (Illumina, San Diego, CA, USA). Barcoded DNA fragments spanning the archaeal and bacterial V4 hypervariable region were amplified with a standardizing level of template DNA (100 pg) to prevent overamplification. These amplicons, generated using adapted primers $533 \mathrm{~F}$ and $806 \mathrm{R}$, were bidirectionally sequenced using the MiSeq system [14]. Preprocessing and classification of sequences was performed using 
Table 1. Patients' characteristics

$\begin{array}{ll}\text { AD patients with no } & \text { AD patients with a confirmed } \\ \text { food allergy }(n=20)\end{array}$

food allergy $(n=62) \quad$ food allergy $(n=20)$

$\begin{array}{lc}\text { Median age, years } & 3.0(0.5-19) \\ \text { Females, } n & 32(52 \%) \\ \text { Median SA-EASI } & 29(2-84) \\ \text { Median TARC, pg/mL } & 1,243(70-10,000) \\ \text { Median total IgE, kU/L } & 62(1.9-4,718) \\ \text { Sensitization to any food allergen, } n & 27(43 \%) \\ \text { On an elimination diet for any food, } n & 19(31 \%) \\ \text { Diagnosed with asthma, } n & 18(29 \%) \\ \text { Diagnosed with rhinoconjunctivitis, } n & 15(24 \%)\end{array}$

$\begin{array}{cr}2.2(0.5-12) & 0.606 \\ 7(35 \%) & 0.196 \\ 46(0-86) & 0.283 \\ 2,251(784-10,000) & 0.013 \\ 564(9-10,601) & <0.001 \\ 20(100 \%) & <0.001 \\ 20(100 \%) & <0.001 \\ 5(25 \%) & 0.727 \\ 5(25 \%) & 0.942\end{array}$

Values in parentheses after median values are min-max. AD, atopic dermatitis; FA, food allergy; SA-EASI, self-administered eczema area and severity index (scored by the research nurse); TARC, thymus and activation regulated chemokine.

modules implemented in the mothur v1.20.0 software platform [15]. The relative abundance of unique sequences was calculated for every fecal sample. The dataset was transformed using a zero mean unit variance transformation for subsequent statistical analyses. The V4 amplicon of the $16 \mathrm{~S}$ rRNA encoding gene allows for the discrimination of several Bifidobacteria species, but not all [16]. Therefore, relevant sequences were blasted in the Ribosomal Database Platform (RDP) to determine a more accurate species level. Shannon diversity indices were calculated to describe the microbial diversity.

\section{Statistical Analysis}

Descriptive statistics were used to describe patient characteristics. Nonparametric tests were used to compare the groups with and without a confirmed food allergy.

\section{Elastic Net Regression}

Bacterial signature species discriminating between the absence and presence of food allergy were selected using elastic net regression. This is a statistical machine-learning approach, applicable to large-scale, structured, and higher-dimensional data. The method is regularization-based and combines the advantages of LASSO regression (sparsity, retaining the feature selection property of reducing coefficients to exact zero values provided by LASSO) and ridge regression (smoothness, a tendency of shrinking coefficients to small values for correlated trending towards each other) [17, 18]. All species present and the correlations between them are taken into account, which allows for the identification of patterns of species rather than individual species [19]. Using elastic net regression, it is not possible to correct for other confounding factors which is common in other types of regression analyses used in medical statistics [19].

Randomization Test and Receiver Operating Characteristics/ Area under the Curve

A randomization test was conducted to test the statistical validity of the results obtained with elastic net regression. ROC/AUC (receiver operating characteristics/area under the curve) scores were generated multiple times after randomly reshuffling the food

Fecal Microbiome and Food Allergy in Pediatric AD allergy diagnoses, while keeping the corresponding microbial profiles intact [20]. The dataset was cross-validated by randomly hiding $30 \%$ of the children from the model and evaluating the prediction quality on that group. The predictive accuracy of the classification model was measured with the ROC/AUC score, using a critical value of 0.05 .

SPSS v22 (IBM, Armonk, NY, USA) was used for descriptive data analysis. GraphPad Prism v6.01 (GraphPad Software, La Jolla, CA, USA) was used for providing graphs and figures. All other statistical analyses were performed using numerical Python v2.7 (Python Software Foundation, https://www.python.org).

\section{Results}

\section{$A D$ and Food Allergy}

We included 82 children in this cross-sectional pilot study. There were no significant differences regarding sex or age between the children who were included and those who were not (data not shown). All 82 children were diagnosed with $\mathrm{AD}, 62$ children had no food allergy, and 20 children had a confirmed food allergy (Table 1). Of the 62 children without food allergy, almost half were sensitized to common food allergens without having symptoms of food allergy after ingestion of the food. In the 20 children with a food allergy, peanut allergy and cow's milk allergy were the most common (Table 2). Multiple food allergies were found in 2 children. On average, a DBPCFC was performed within 10 months (range 1-27 months) of providing the fecal samples.

\section{Sequence and Microbiota Characteristics}

A total of 2,609,478 high-quality sequences were obtained (mean 27,182; range 5,825-105,404 sequences/ 
Table 2. The number of children with confirmed food allergies

\begin{tabular}{|c|c|c|c|c|}
\hline & A confirmed $\mathrm{FA}^{\mathrm{a}}$ & A positive $\mathrm{DBPCFC}^{\mathrm{b}}$ & $\begin{array}{l}\text { An obvious clinical } \\
\text { history (only) }\end{array}$ & $\begin{array}{l}\text { FA was predicted, based on elevated } \\
\text { sIgE to Ara h } 2(>5.17 \mathrm{kU} / \mathrm{L})^{\mathrm{c}}\end{array}$ \\
\hline Peanut & $8(40 \%)$ & $3(15 \%)$ & $1(5 \%)$ & $4(20 \%)$ \\
\hline Cow's milk & $8(40 \%)$ & $6(30 \%)$ & $2(10 \%)$ & \\
\hline Hen's egg & $4(20 \%)$ & $3(15 \%)$ & $1(5 \%)$ & \\
\hline Pistachio & & & $1(5 \%)$ & \\
\hline Soy & 0 & & & \\
\hline Fish/shrimp & 0 & & & \\
\hline Total & $20(100 \%)$ & & & \\
\hline
\end{tabular}

FA, food allergy. ${ }^{\text {a }}$ Multiple FAs resulted in multiple entries: 1 patient had a confirmed FA to cow's milk, peanut, and hen's egg; 1 patient had a confirmed FA to hazelnut and hen's egg. ${ }^{b}$ Including late reactions. ${ }^{c}$ According to Klemans et al. [11].

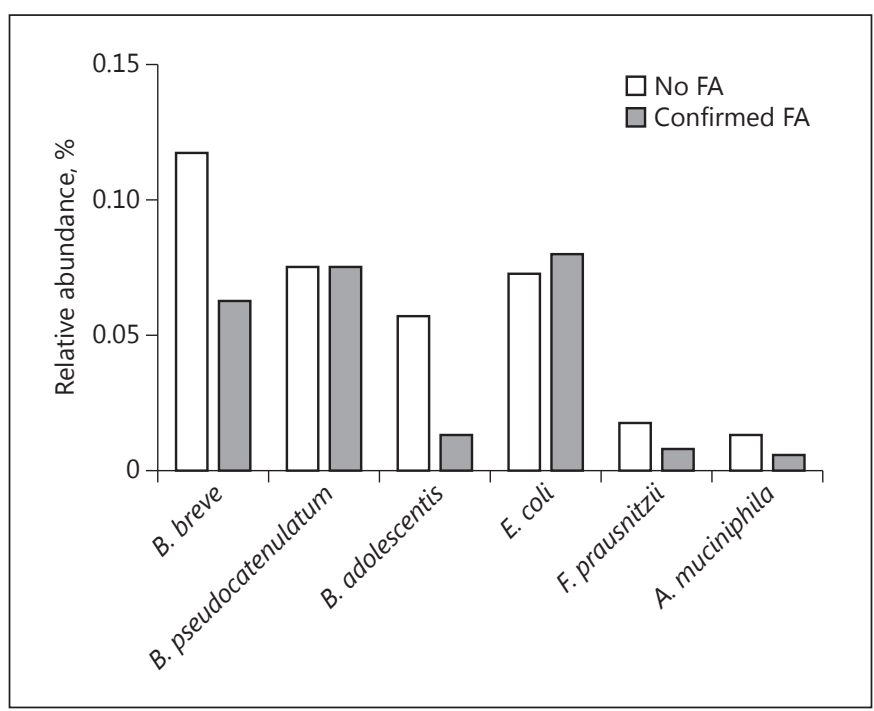

Fig. 1. Relative abundance of the microbial signature species in children with $\mathrm{AD}$, without $\mathrm{AD}$, and with a confirmed food allergy (FA).

sample) that could be assigned to 12 different phyla and 1,000 unique sequences. The most predominant phyla, based on mean relative abundance, were Firmicutes (47\%), Actinobacteria (32\%), Bacteroidetes (9\%), Proteobacteria (8\%), and Verrucomicrobia (2\%), characteristic for the gut microbiome of children [21]. Predominant families were Bifidobacteriaceae (28\%), Lachnospiraceae (27\%), Ruminococcaceae (10\%), Enterobacteriaceae
(5\%), Streptococcaceae (4\%), and Coriobacteriaceae (3.5\%). Median Shannon diversity indices calculated for the group of children with and without a food allergy were 3.61 (IQR 1.16) and 3.93 (IQR 1.09), respectively $(p=0.430)$.

\section{Identification of Microbial Biomarkers Related to \\ Food Allergy}

We identified 6 microbial species from 4 families that, together, discriminate between the absence and presence of food allergy in children with AD: Bifidobacterium breve, Bifidobacterium pseudocatenulatum, Bifidobacterium adolescentis (Bifidobacteriaceae), Escherichia coli (Enterobacteriaceae), Faecalibacterium prausnitzii (Ruminococcaceae), and Akkermansia muciniphila (Verrucomicrobiaceae). On the species level, $B$. breve/longum and B. pseudocatenulatum/catenulatum/gallicum/ kashiwanohense could not be distinguished after additional blasting in the RDP, and are referred to as B. breve and B. pseudocatenulatum throughout the paper.

Figure 1 shows the relative abundance of the 6 identified signature species. The fecal microbiome of children with $\mathrm{AD}$ and food allergy harbored relatively more $E$. coli and $B$. pseudocatenulatum, and less B. breve, B. adolescentis, F. prausnitzii, and A. muciniphila than children with $\mathrm{AD}$ without food allergy. The randomization test indicates that the combination of these 6 species is significantly different in the 2 groups $(p=0.001)$, even though the relative abundance of some single species may seem
80

Int Arch Allergy Immunol 2018;175:77-84 DOI: $10.1159 / 000484897$
Fieten/Totté/Levin/Reyman/Meijer/ Knulst/Schuren/Pasmans 


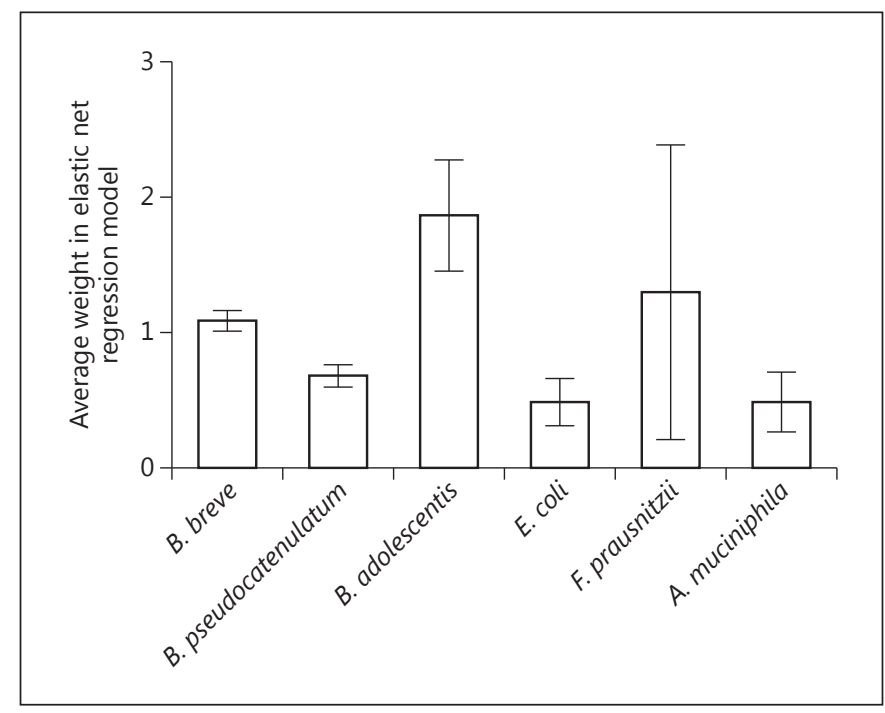

Fig. 2. Importance index for signature species in the elastic net regression model.

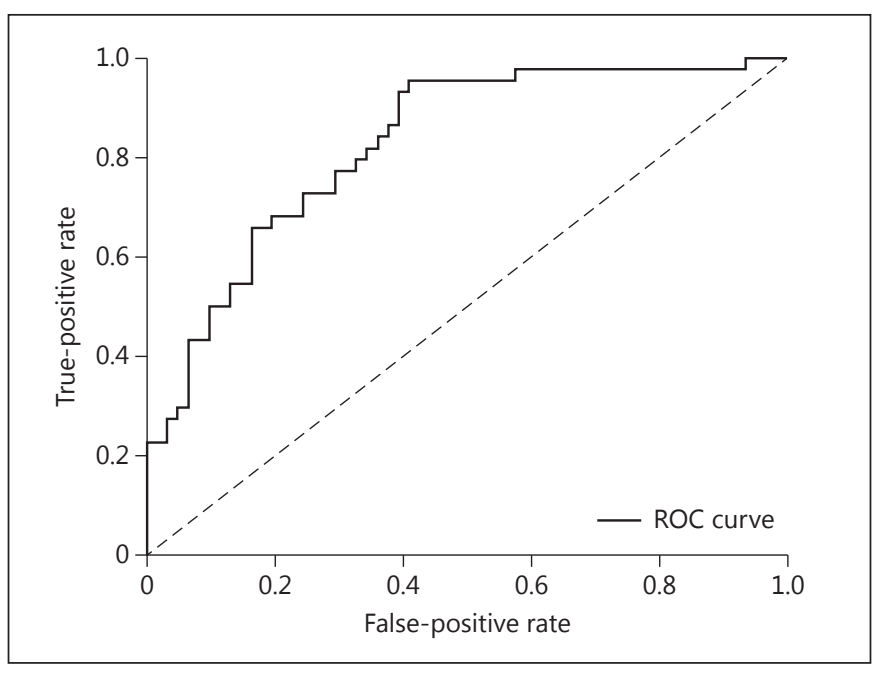

Fig. 3. Receiver operating characteristic (ROC) curve of the elastic net regression model. The area under the curve is 0.83 .

similar on a group level (Fig. 1). Different relative contributions from the single species towards the total distinctive properties are distinguished, with B. breve, B. adolescentis, and $F$. prausnitzii having a greater influence than B. pseudocatenulatum, E. coli, and A. muciniphila, expressed as importance indices based on the elastic net regression (Fig. 2). The overall predictive accuracy of the classification model (AUC) is 0.83 (Fig. 3), with a sensitivity of 0.77 and a specificity of 0.80 . Online supplementary Figures S1-S3 (for all online suppl. material, see

Fecal Microbiome and Food Allergy in Pediatric AD www.karger.com/doi/10.1159/000484897) show the relative abundance of the signature species, the distribution of the 30 most abundant species, and the individual distribution of the signature species.

\section{Discussion}

We analyzed the fecal microbiome of children with $\mathrm{AD}$ with or without a concomitant food allergy, and found that a combination of 6 microbial species, including E. coli, F. prausnitzii, A. muciniphila, and 3 types of Bifidobacteria, discriminates between the presence and absence of food allergy in children with $\mathrm{AD}(p=0.001)$. The fecal microbiome of children with $\mathrm{AD}$ and food allergy harbored relatively more E. coli and B. pseudocatenulatum, and less B. breve, $B$. adolescentis, F. prausnit$z i i$, and $A$. muciniphila than that of children with $\mathrm{AD}$ without food allergy. We found no differences in microbial diversity (according to the Shannon index) between the children with and without food allergy.

This is the first pilot study that identifies microbial signatures specific for food allergy in a group of children with $\mathrm{AD}$ by making use of $16 \mathrm{~S}$ rRNA sequencing techniques to generate unique sequences, followed by statistical machine-learning approaches. Previous studies mainly used culture-based techniques to analyze the intestinal microbiome or used 16S rRNA sequencing techniques, but subsequently simplified the data in the analysis stage, by focusing on key groups of species or analyzing the data on a family or genus level. However, this approach leads to less detailed information. For example, the identification of B. pseudocatenulatum, B. breve, and B. adolescentis would not have been possible when analyzing the data on a family level. Furthermore, the elastic net regression model takes group-based species interactions into account. Since interactions between species in the gut microbiome occur, this approach may lead to biologically more reliable results than with other statistical regression approaches [22].

Our study demonstrates that children with $\mathrm{AD}$ and a food allergy had significantly less $F$. prausnitzii and $A$. muciniphila than children with $\mathrm{AD}$ without a food allergy. F. prausnitzii and A. muciniphila have been gaining interest more recently because of their immune-modulatory properties and possible role in mucosal tolerance. $F$. prausnitzii is the most common abundant species in the human intestinal microbiome. Its decreased abundance has been associated with several diseases, including allergic disease and $\mathrm{AD}$ [23-25]. F. prausnitzii is the main pro- 
ducer of butyrate in the colon, an energy source for colonocytes with important anti-inflammatory effects. It also secretes anti-inflammatory molecules that directly modulate the host immune system, stimulates IL10-producing regulatory $\mathrm{T}$ cells and is involved in the balance between effector and regulatory $\mathrm{T}$ cells $[26,27]$. A. $m u$ ciniphila is also involved in the immunological homeostasis of the gut mucosa and gut barrier function, via an outer membrane protein that stimulates IL10 production [28].

Bifidobacteria and E. coli have been associated with food allergy and $\mathrm{AD}$ in other studies [29]. Less Bifidobacteria in the feces of children with a confirmed cow's milk allergy has been reported [30]. Cow's milk allergy was a common food allergy in our study population, so it is possible that our results regarding $B$. breve and B. adolescentis were mainly contributed by the children allergic to cow's milk. Furthermore, we found an increased relative abundance of E. coli in the food-allergic group. E. coli has previously been associated with the diagnosis of $\mathrm{AD}$, with increasing numbers of $E$. coli further increasing this risk [31]. The children in our study were all diagnosed with $\mathrm{AD}$ with varying severity. However, the higher levels of thymus and activation regulated chemokine (TARC) in the food-allergic group suggest increased AD severity compared to the nonallergic group. This raises the possibility that the selected biomarkers also correlated with AD severity, which fits with the observation that the prevalence of food allergy is higher in children with greater disease severity [32].

All the microbial species resulting from our analysis have previously been correlated with atopic disease in other studies. This might raise the question of whether we are looking at a food allergy-specific microbial profile or a profile that is related to atopic diseases in general, as most of these children have or will develop other comorbidities within the atopic syndrome. Atopic disease has been defined differently in previous studies. In our study, all children were clinically diagnosed with $\mathrm{AD}$ and, in addition, asthma and allergic rhinitis were confirmed or ruled out based on the child's clinical history. Food allergy was diagnosed based on DCPCFC in the majority of patients. Post hoc analyses showed no significant differences between the group with and the group without food allergy with regard to other atopic diagnoses, suggesting that the species identified species indicate food allergy rather than general atopy.

Our study supports the hypothesis that, in children with $\mathrm{AD}$, the intestinal microbiome differs in children with and without food allergy. Intestinal microbiota regu- late the development of functions of a diverse range of $\mathrm{T}$ cells, such as Th17, Th1, Th2, and regulatory T cells, and also modulate innate lymphoid cells $[33,34]$. By modifying the response of gut-associated lymphoid tissues, intestinal microbiota may influence the development of oral tolerance [35]. A recent study on humans showed that delayed colonization with Bacteroidetes is associated with a poorly developed Th1 response, which is important in immune tolerance [36]. It is also possible that disruption of the gut microbiome alters the epithelial integrity of the gut, thereby increasing the risk of allergic sensitization through the direct uptake of allergens [7]. However, the exact mechanisms by which the intestinal microbiome influences food allergy are not elucidated yet. Furthermore, it is not clear whether a change in the microbiome precedes or follows the development of food allergy.

Long-term dietary intake affects gut microbiome composition, together with host genetics, age, medication, and general lifestyle [37]. Our study population consumed a Western diet. In addition to this, established food allergies lead to an elimination diet, where the specific food allergen is excluded from the general diet. We cannot exclude the possibility that an elimination diet where one particular food is excluded from the diet would also leads to detectable changes in the fecal microbial composition, as has been demonstrated where there is an increased consumption of specific foods [38]. However, in our study, one-third of the children in the group without food allergy also reported being on an elimination diet for a specific food, for various reasons. Furthermore, dietary intake varies according to personal preference. Therefore, it is unlikely that the observed microbial differences can be solely attributed to the dietary differences. Besides a self-reported elimination diet, dietary intake was not further assessed in this study because it is very difficult to assess accurately.

A limitation of our cross-sectional study was the heterogeneity of the study population. All of the children were diagnosed with $\mathrm{AD}$, with varying disease severity, age, and food allergies. We did not include any healthy controls. As was to be expected in children, cow's milk allergy and peanut allergy were the most common food allergies in our study population, so it is possible that our results were influenced by the contribution of these particular food allergies. It is also plausible that distinct microbes are associated with different food allergies [29]. Due to a lack of statistical power, we were unable to select signature species for specific food allergies. Variables that are known to influence the gut microbiome, such as the
82

Int Arch Allergy Immunol 2018;175:77-84 DOI: $10.1159 / 000484897$
Fieten/Totté/Levin/Reyman/Meijer/ Knulst/Schuren/Pasmans 
use of antibiotics, birth via caesarean section, or breastfeeding, were not assessed in this study [7]. Furthermore, because of the time between the acquisition of the fecal sample and the DBPCFC, transient food allergies could have resulted in the misclassification of some children with cow's milk allergy and hen's egg allergy.

Our findings are based on a study population of children with $\mathrm{AD}$ from an academic center. Identifying the microbes that are related to food allergy may help in the development of future interventions. However, future studies are needed to confirm our findings in the community, preferably with prospective study designs using welldefined patient populations to further explore the potential of the fecal microbial colonization patterns associated with specific food allergies in children with AD. Control groups should also be included, i.e., children with food allergy without $\mathrm{AD}$, or with severe "extrinsic" $\mathrm{AD}$ without food allergy. These groups should be of sufficient size to allow for the stratification of different food allergies.

\section{Conclusion}

In this pilot study, we identified a microbial signature in children with $\mathrm{AD}$ that discriminates between the absence and presence of food allergy. Future studies are needed to confirm our findings.

\section{Acknowledgements}

We acknowledge Ms. J. Beutler and Ms. A. Ouwens for their technical assistance. Current affiliations of S.G.M.A.P.: Department of (Pediatric) Dermatology, Sophia Children's Hospital, Erasmus MC University Medical Center Rotterdam, Rotterdam, The Netherlands.

\section{Disclosure Statement}

The authors have no conflicts of interest to declare.

\section{References}

1 Asher MI, Montefort S, Bjorksten B, Lai CK, Strachan DP, Weiland SK, Williams H: Worldwide time trends in the prevalence of symptoms of asthma, allergic rhinoconjunctivitis, and eczema in childhood: ISAAC phases I and III repeat multicountry cross-sectional surveys. Lancet 2006;368:733-743.

2 Alfven T, Braun-Fahrlander C, Brunekreef B, von Mutius E, Riedler J, Scheynius A, van Hage M, Wickman M, Benz MR, Budde J, Michels KB, Schram D, Ublagger E, Waser M, Pershagen G: Allergic diseases and atopic sensitization in children related to farming and anthroposophic lifestyle - the PARSIFAL study. Allergy 2006;61:414-421.

3 Ege MJ, Mayer M, Normand AC, Genuneit J, Cookson WO, Braun-Fahrlander C, Heederik D, Piarroux R, von Mutius E: Exposure to environmental microorganisms and childhood asthma. N Engl J Med 2011;364:701-709.

4 Genuneit J, Strachan DP, Buchele G, Weber J, Loss G, Sozanska B, Boznanski A, Horak E, Heederik D, Braun-Fahrlander C, von Mutius E: The combined effects of family size and farm exposure on childhood hay fever and atopy. Pediatr Allergy Immunol 2013;24:293298.

5 Hooper LV, Littman DR, Macpherson AJ: Interactions between the microbiota and the immune system. Science 2012;336:12681273.

6 Wold AE: The hygiene hypothesis revised: is the rising frequency of allergy due to changes in the intestinal flora? Allergy 1998;53:20-25.
7 Molloy J, Allen K, Collier F, Tang ML, Ward AC, Vuillermin P: The potential link between gut microbiota and IgE-mediated food allergy in early life. Int J Environ Res Public Health 2013;10:7235-7256.

8 Sankar SA, Lagier JC, Pontarotti P, Raoult D, Fournier PE: The human gut microbiome, a taxonomic conundrum. Syst Appl Microbiol 2015;38:276-286

9 Williams HC, Burney PG, Hay RJ, Archer CB, Shipley MJ, Hunter JJ, Bingham EA, Finlay AY, Pembroke AC, Graham-Brown RA: The UK Working Party's Diagnostic Criteria for Atopic Dermatitis. I. Derivation of a minimum set of discriminators for atopic dermatitis. Br J Dermatol 1994;131:383396.

10 Housman TS, Patel MJ, Camacho F, Feldman SR, Fleischer AB Jr, Balkrishnan R: Use of the self-administered eczema area and severity index by parent caregivers: results of a validation study. Br J Dermatol 2002;147:11921198.

11 Klemans RJ, Otte D, Knol M, Knol EF, Meijer Y, Gmelig-Meyling FH, Bruijnzeel-Koomen CA, Knulst AC, Pasmans SG: The diagnostic value of specific IgE to Ara $\mathrm{h} 2$ to predict peanut allergy in children is comparable to a validated and updated diagnostic prediction model. J Allergy Clin Immunol 2013;131: 157-163.

12 Sampson HA, Gerth van WR, Bindslev-Jensen C, Sicherer S, Teuber SS, Burks AW, Dubois AE, Beyer K, Eigenmann PA, Spergel JM,
Werfel T, Chinchilli VM: Standardizing double-blind, placebo-controlled oral food challenges: American Academy of Allergy, Asthma \& Immunology-European Academy of Allergy and Clinical Immunology PRACTALL consensus report. J Allergy ClinImmunol 2012;130:1260-1274.

13 Bogaert D, Keijser B, Huse S, Rossen J, Veenhoven R, van GE, Bruin J, Montijn R, Bonten M, Sanders E: Variability and diversity of nasopharyngeal microbiota in children: a metagenomic analysis. PLoS One 2011;6: e17035.

14 Caporaso JG, Lauber CL, Walters WA, BergLyons D, Huntley J, Fierer N, Owens SM, Betley J, Fraser L, Bauer M, Gormley N, Gilbert JA, Smith G, Knight R: Ultra-high-throughput microbial community analysis on the Illumina HiSeq and MiSeq platforms. ISMEJ 2012;6:1621-1624.

15 Schloss PD, Westcott SL, Ryabin T, Hall JR, Hartmann M, Hollister EB, Lesniewski RA, Oakley BB, Parks DH, Robinson CJ, Sahl JW, Stres B, Thallinger GG, Van Horn DJ, Weber CF: Introducing mothur: open-source, platform-independent, community-supported software for describing and comparing microbial communities. Appl Environ Microbiol 2009;75:7537-7541.

16 Wang Q, Garrity GM, Tiedje JM, Cole JR: Naive Bayesian classifier for rapid assignment of rRNA sequences into the new bacterial taxonomy. Appl Environ Microbiol 2007;73: 5261-5267.
Fecal Microbiome and Food Allergy in Pediatric AD
Int Arch Allergy Immunol 2018;175:77-84 DOI: 10.1159/000484897 
17 Tibshirani R: Regression shrinkage and selection via the LASSO. J R Stat Soc Series B Stat Methodol 1996:267-288.

18 Guyon I, Elisseeff A: An introduction to variable and feature selection. J Mach Learn Res 2003;3:1157-1182.

19 Zou H, Hastie T: Regularization and variable selection via the elastic net. J R Stat Soc Series B Stat Methodol 2005;67:301-320.

20 Fawcett T: An introduction to ROC analysis Pattern Recognit Lett 2006;27:861-874.

21 Bergstrom A, Skov TH, Bahl MI, Roager HM, Christensen LB, Ejlerskov KT, Molgaard C, Michaelsen KF, Licht TR: Establishment of intestinal microbiota during early life: a longitudinal, explorative study of a large cohort of Danish infants. Appl Environ Microbiol 2014;80:2889-2900.

22 Faust K, Sathirapongsasuti JF, Izard J, Segata N, Gevers D, Raes J, Huttenhower C: Microbial co-occurrence relationships in the human microbiome. PLoS Comput Biol 2012; 8:e1002606.

23 Candela M, Rampelli S, Turroni S, Severgnini M, Consolandi C, De BG, Masetti R, Ricci G, Pession A, Brigidi P: Unbalance of intestinal microbiota in atopic children. BMC Microbiol 2012;12:95.

24 Miquel S, Martin R, Rossi O, Bermudez-Humaran LG, Chatel JM, Sokol H, Thomas M, Wells JM, Langella P: Faecalibacterium prausnitzii and human intestinal health. Curr Opin Microbiol 2013;16:255-261.

25 Song H, Yoo Y, Hwang J, Na YC, Kim HS: Faecalibacterium prausnitzii subspecies-level dysbiosis in the human gut microbiome underlying atopic dermatitis. J Allergy Clin Immunol 2016;137:852-860.
26 Sokol H, Pigneur B, Watterlot L, Lakhdari O, Bermudez-Humaran LG, Gratadoux JJ, Blugeon S, Bridonneau C, Furet JP, Corthier G, Grangette C, Vasquez N, Pochart P, Trugnan G, Thomas G, Blottiere HM, Dore J, Marteau P, Seksik P, Langella P: Faecalibacterium prausnitzii is an anti-inflammatory commensal bacterium identified by gut microbiota analysis of Crohn disease patients. Proc Natl Acad Sci USA 2008;105:16731-16736.

27 Rossi O, van Berkel LA, Chain F, Tanweer Khan M, Taverne N, Sokol H, Duncan SH, Flint HJ, Harmsen HJ, Langella P, Samsom JN, Wells JM: Faecalibacterium prausnitzii a2-165 has a high capacity to induce IL-10 in human and murine dendritic cells and modulates T cell responses. Sci Rep 2016;6:18507.

28 Ottman N, Reunanen J, Meijerink M, Pietila TE, Kainulainen V, Klievink J, Huuskonen L, Aalvink S, Skurnik M, Boeren S, Satokari R, Mercenier A, Palva A, Smidt H, de Vos WM, Belzer C: Pili-like proteins of Akkermansia muciniphila modulate host immune responses and gut barrier function. PLoS One 2017; 12:e0173004.

29 Huang YJ, Marsland BJ, Bunyavanich S, O’Mahony L, Leung DY, Muraro A, Fleisher TA: The microbiome in allergic disease: current understanding and future opportunities - 2017 PRACTALL document of the American Academy of Allergy, Asthma \& Immunology and the European Academy of Allergy and Clinical Immunology. J Allergy Clin Immunol 2017;139:1099-1110.

30 Thompson-Chagoyan OC, Vieites JM, Maldonado J, Edwards C, Gil A: Changes in faecal microbiota of infants with cow's milk protein allergy - a Spanish prospective case-control 6-month follow-up study. Pediatr Allergy Immunol 2010;21:e394-e400.
31 Penders J, Thijs C, van den Brandt PA, Kummeling I, Snijders B, Stelma F, Adams H, van Ree R, Stobberingh EE: Gut microbiota composition and development of atopic manifestations in infancy: the KOALA birth cohort study. Gut 2007;56.

32 Silverberg JI, Simpson EL: Association between severe eczema in children and multiple comorbid conditions and increased healthcare utilization. Pediatr Allergy Immunol 2013;24:476-486.

33 Round JL, Mazmanian SK: The gut microbiota shapes intestinal immune responses during health and disease. Nat Rev Immunol 2009;9: 313-323.

34 Rachid R, Chatila TA: The role of the gut microbiota in food allergy. Curr Opin Pediatr 2016;28:748-753.

35 Nowak-Wegrzyn A, Szajewska H, Lack G: Food allergy and the gut. Nat Rev Gastroenterol Hepatol 2017;14:241-257.

36 Jakobsson HE, Abrahamsson TR, Jenmalm MC, Harris K, Quince C, Jernberg C, Bjorksten B, Engstrand L, Andersson AF: Decreased gut microbiota diversity, delayed Bacteroidetes colonisation and reduced Th1 responses in infants delivered by caesarean section. Gut 2014;63:559-566.

37 De Filippo C, Cavalieri D, DiPaola M, Ramazzotti M, Poullet JB, Massart S, Collini S, Pieraccini G, Lionetti P: Impact of diet in shaping gut microbiota revealed by a comparative study in children from Europe and rural Africa. Proc Natl Acad Sci USA 2010;107: 14691-14696.

38 Graf D, Di Cagno R, Fak F, Flint HJ, Nyman M, Saarela M, Watzl B: Contribution of diet to the composition of the human gut microbiota. Microb Ecol Health Dis 2015;26:26164. 